\title{
Improving preoperative detection of synchronous liver metastases in pancreatic cancer with combined contrast-enhanced and diffusion-weighted MRI
}

\author{
D. M. Riviere ${ }^{1}$ - E. J. M. van Geenen ${ }^{2}$ - B. M. van der Kolk ${ }^{3}$ I. D. Nagtegaal ${ }^{4}$. S. A. Radema ${ }^{5}$. \\ C. J. H. M. van Laarhoven ${ }^{3}$ J. J. Hermans ${ }^{1}$
}

Published online: 18 January 2019

(c) The Author(s) 2019

\begin{abstract}
Purpose To explore the value of gadolinium-enhanced MRI combined with diffusion-weighted MRI (Gd-enhanced MRI with DWI) in addition to contrast-enhanced CT (CECT) for detection of synchronous liver metastases for potentially resectable pancreatic cancer.

Methods By means of a retrospective cohort study we included patients with potentially resectable pancreatic cancer on CECT, who underwent Gd-enhanced MRI with DWI between January 2012 and December 2016. A single observer evaluated MRI and CT and was blinded to imaging, pathology, and surgery reports. Liver lesions were scored in both modalities, using a 3-point scale: 1-benign, 2-indeterminate, 3- malignant (i.e., metastasis). The primary outcome parameters were the presence of liver metastases on Gd-enhanced MRI with DWI and the sensitivity of Gd-enhanced MRI with DWI for synchronous liver metastases.

Results We included 66 patients (42 men, 24 women; median age 65 years, range 36-82 years). In 19 patients, liver metastases were present, which were confirmed by histopathology $(n=12),{ }^{18}$ FDG-PET $(n=6)$, or surgical inspection $(n=1)$. Gd-enhanced MRI with DWI showed metastases in $16 / 19$ patients $(24 \%)$, which resulted in a sensitivity of $84 \%$ (95\% CI 60-97\%). Contrast-enhanced MRI showed 156 and DWI 397 metastases $(p=0.051)$, and 339 were particularly small $(<5 \mathrm{~mm})$.

Conclusions In this study, Gd-enhanced MRI with DWI detected synchronous liver metastases in $24 \%$ of patients with potentially resectable pancreatic cancer on CECT with a sensitivity of $84 \%$. Diffusion-weighted MRI showed a greater number of metastases than any other sequence, particularly small metastases $(<5 \mathrm{~mm})$.
\end{abstract}

Keywords Pancreatic cancer $\cdot$ Pancreatic ductal carcinoma $\cdot$ Diffusion-weighted MRI $\cdot$ Metastases $\cdot$ Liver

J. J. Hermans

John.Hermans@radboudumc.nl

1 Department of Radiology and Nuclear Medicine, Radboud University Medical Center, Nijmegen, NL, The Netherlands

2 Department of Gastroenterology, Radboud University Medical Center, Nijmegen, NL, The Netherlands

3 Department of Surgery, Radboud University Medical Center, Nijmegen, NL, The Netherlands

4 Department of Pathology, Radboud University Medical Center, Nijmegen, NL, The Netherlands

5 Department of Oncology, Radboud University Medical Center, Nijmegen, NL, The Netherlands

\section{Introduction}

Pancreatic cancer is one of the most lethal forms of cancer with a 5-year relative survival rate of $6 \%$ reported by the American Cancer Society [1]. Total deaths due to pancreatic cancer are increasing dramatically and expected to become the second leading cause of cancer-related deaths before $2030[2,3]$. Surgery of localized pancreatic cancer offers the only realistic chance to cure. Approximately $10-20 \%$ of patients do have unexpected liver metastases, peritoneal carcinomatosis, or locally advanced disease at the time of surgery [4-6]. More than 50\% of all liver metastases develop in the first six months postoperatively, even in patients with early tumor stage [7]. These findings 
suggest that liver metastases are already present at the time of surgery, which is supported by the mathematical model by Haeno et al., predicting that patients likely harbor metastases at diagnosis [8]. These synchronous liver metastases are not identified pre-operatively, as they are too small to be detected by routine preoperative ultrasound and contrast-enhanced CT (CECT) [9].

International guidelines advise CECT for routine diagnosing and staging of pancreatic cancer, whereas MRI is mostly used for characterization of indeterminate liver lesions [10]. CECT allows accurate assessment of the relationship between the tumor and critical arterial and venous structures [11]. However, the detection of subcentimeter metastases by CECT poses a greater challenge. Even if subcentimeter liver lesions are identified on a preoperative CT scan, the ability to precisely characterize those lesions as malignant is limited [12].

Nowadays, diffusion-weighted MR imaging (DWI) is increasingly used for hepatic imaging and has been shown to be a valuable tool in both detection and characterization of focal liver lesions with a sensitivity ranging from 86 to $97 \%$ and 60 to $91 \%$ for subcentimeter lesions [13-16]. Most studies have been performed for liver metastases of colorectal cancer. There are limited studies performed in pancreatic cancer, all concluding that additional MRI is useful in detecting liver metastases. Most studies used 1,5T scanners [9, 17-20]. In the 3,0T scanners, the increased signal-to-noise ratio can be translated into a higher resolution, and the improved contrast-to-noise ratio of gadolinium-based contrast agent can both contribute to improved lesion detection and characterization [21]. Liverspecific contrast agent was used in the studies by Ito et al., Motosugi et al. and Chew et al. for the detection of liver metastases [17, 19, 22]. In the ESGAR consensus statement, gadoxetate disodium is recommended for the diagnosis and characterization of malignant liver lesions in non-cirrhotic livers [23]. Aside from the associated higher costs, the relative hepatic enhancement could be negatively influenced by high serum bilirubin levels, which is common in patients with obstructive jaundice in pancreatic cancer of the head [24]. In this retrospective study, we evaluated the sensitivity of nonspecific extracellular gadolinium contrast-enhanced MRI (Gd-enhanced MRI) combined with DWI for synchronous liver metastases in potentially resectable pancreatic cancer on a $3 \mathrm{~T}$ MR scanner.

\section{Materials and methods}

All procedures performed in studies involving human participants were in accordance with the ethical standards of the institutional research committee and with the 1964
Helsinki declaration and its later amendments or comparable ethical standards. For this type of study formal consent is not required.

\section{Setting and participants}

All patients older than 18 years with potentially resectable pancreatic cancer without liver metastases on CECT and additional Gd-enhanced MRI with DWI performed in our hospital from January 2012 to December 2016 were eligible for inclusion. Patients were recruited from the Radiology Information System. MRI was routinely performed in our center in all patients with potentially resectable disease or indeterminate liver lesions on CECT. Patients with locally resectable or borderline resectable pancreatic cancer were included. Resectability was established using criteria of the Dutch Pancreatic Cancer Group (PREOPANC trial, DPCG 2012). Exclusion criteria were local or systemic treatment for pancreatic cancer prior to imaging, locally advanced pancreatic cancer on CECT, incomplete liver imaging, and a time interval between CT and MRI or imaging and surgery of more than 2 months. The primary outcome parameters were the presence of liver metastases on Gd-enhanced MRI with DWI and the sensitivity of Gd-enhanced MRI with DWI for synchronous liver metastases. The secondary endpoint was the number of lesions suspicious for metastases detected by the different MRI sequences. Confirmation of liver metastases was obtained by histopathology, ${ }^{18}$ FDGPET, and surgical findings. Explorative surgery was performed in all patients with (borderline) resectable tumors without histopathological proof or ${ }^{18}$ FDG-PET confirmation of metastases. Demographic characteristics were collected from the electronic medical records. Survival rates were obtained from the general practitioners in October 2015 and were updated in January 2018 from data in the electronic medical records.

\section{CT technique}

CECT was performed in different hospitals and produced at different models of 16- and 64-row multidetector CT scanners. Only high-quality datasets with image acquisition in the portal-venous phase and slice thickness of 3-5 mm were included for analysis.

\section{MRI technique}

All MR imaging of the abdomen was performed in our academic tertiary referral center on a 3.0 Tesla system (Magnetom Skyra, Siemens Healthcare, Erlangen, Germany). The imaging protocol is displayed in Table 1 . The protocol consisted of a T1-weighted axial in- and opposed 
Table 1 MR imaging parameters

\begin{tabular}{|c|c|c|c|c|c|c|}
\hline \multirow{2}{*}{$\begin{array}{l}\text { Parameter } \\
\text { Plane }\end{array}$} & \multirow{2}{*}{$\begin{array}{l}\text { T1-weighted imaging in- } \\
\text { and opposed phase (VIBE) } \\
\text { Axial }\end{array}$} & \multicolumn{2}{|c|}{ T2-weighted imaging (HASTE) } & \multicolumn{2}{|c|}{$\begin{array}{l}\text { T1-weighted imaging } \\
\text { (VIBE) pre- and post-contrast }\end{array}$} & \multirow{2}{*}{$\begin{array}{l}\text { Diffusion-weighted } \\
\text { imaging (SPAIR) } \\
\text { Axial }\end{array}$} \\
\hline & & Axial & Coronal & Axial & Coronal & \\
\hline Section thickness (mm) & 3 & 5 & & 3 & 1.5 & 5 \\
\hline Intersection gap (mm) & 0 & 0.5 & & 0 & 0 & 1 \\
\hline Repetition time (msec) & 4.35 & 1600 & 1400 & 4.34 & 2.92 & $>2100$ \\
\hline Echo time (msec) & $2.45-1.33$ & 95 & 87 & 1.89 & 1.05 & 71 \\
\hline Flip angle (degree) & 9 & $90 / 160$ & $90 / 180$ & 9 & 11 & $90 / 180$ \\
\hline Field of view $(\mathrm{cm})$ & 30 & 35 & & 30 & 30 & 38 \\
\hline Matrix & $320 \times 195$ & $320 \times 256$ & & $320 \times 195$ & $256 \times$ & $192 \times 156$ \\
\hline Bandwidth (Hz/pix) & 975 & $710 / 710$ & & 445 & 650 & 1736 \\
\hline
\end{tabular}

phase gradient-echo VIBE, a half Fourier acquisition single-shot turbo spin-echo (HASTE), pre- and post-contrast T1-weighted 3D gradient-echo VIBE, and a respiratory triggered single-shot spin-echo echoplanar DWI in the transverse plane with monopolar diffusion gradients along three orthogonal directions with b-values of $0 / 50,500$, and $800 \mathrm{~s} / \mathrm{mm}^{2}$, using $\delta=10.1 \mathrm{~ms}$ and $\Delta=33.5 \mathrm{~ms}$. Fifteen $\mathrm{ml}$ of gadoterate meglumine $0.5 \mathrm{mmol} / \mathrm{mL}$ (Dotarem, Guerbet, Villepinte, France) was injected in an antecubital vein at $2.5 \mathrm{ml} / \mathrm{s}$ with a saline flush $(\mathrm{NaCl} 0.9 \%)$ of $20 \mathrm{ml}$ at $2.5 \mathrm{ml} / \mathrm{s}$ using a pump injector (Optistar Elite, Mallinckrodt, Dublin, Ireland). MR cholangiopancreatographic images were also obtained; these images were not used in this study.

\section{Image interpretation}

MR images were consecutively reviewed by a radiologist (JH) with 14 years of experience in abdominal and pancreas imaging, on a commercial PACS workstation (Impax, Agfa Healthcare, Belgium). The observer was blinded to all clinical information, pathology reports, and the original radiology report, aside from the diagnosis of pancreatic cancer. In both modalities, liver lesions were scored using a 3-point scale: 1-benign, 2-indeterminate, 3-malignant (i.e., metastasis). Number, size, location, and imaging characteristics and the presumed diagnosis of the lesion were noted. Benign lesions were diagnosed using established imaging criteria [25-27]. On CECT, hypodense lesions that show typical features of a simple cyst (fluid attenuation measurements, round-oval, well-defined borders, no contrast enhancement), a hemangioma (localization next to vessels, peripheral nodular enhancement, centripetal fillin), or focal fatty infiltration (geographic hypodense area, angular margins, typical location) are classified as benign lesions. Indeterminate liver lesions on CECT included hypodense liver lesions that were too small to be characterized. Metastases are hypodense lesions with rim enhancement. On MRI, metastases of pancreatic cancer are typically of moderately high to isointense signal intensity on T2W-images and mildly hypointense to isointense on T1W-images. Metastases can either be hypo- or hypervascular, and show homogeneous or peripheral enhancement (ring or wedge-shaped) in the arterial phase, homogeneous enhancement or peripheral enhancement with complete or incomplete centripetal progression in the portal-venous and interstitial phase [28]. On DWI, a lesion was classified as malignant (i.e., metastasis) when it was (moderately) hyperintense at $b=0 / 50 \mathrm{~s} / \mathrm{mm}^{2}$ and remained hyperintense at the highest $b=800 \mathrm{~s} / \mathrm{mm}^{2}$ and a lesion was considered benign when it was hyperintense at $b=0 / 50 \mathrm{~s} /$ $\mathrm{mm}^{2}$ and showed a substantial decrease in signal intensity at higher b-values $\left(b=500\right.$ and $\left.800 \mathrm{~s} / \mathrm{mm}^{2}\right)$. If none of the criteria were met, a lesion was classified as indeterminate. For the analysis, indeterminate lesions were classified as benign, as in clinical practice indeterminate lesions that cannot be further classified will be regarded as benign unless proven otherwise by biopsy. Whenever more than ten malignant lesions (i.e., metastasis) per slice were present, the number of malignant lesions per slice was estimated in dozens.

\section{Statistical methods}

All data were processed using SPSS software (version 20, SPSS, Chicago, IL). The sensitivity of Gd-enhanced MRI with DWI was calculated with a $95 \%$ confidence interval (CI). ANOVA test was performed to determine the differences between the group with liver metastases and the group without liver metastases. Paired samples $t$ test was used to determine the difference between contrast-enhanced MRI and DWI regarding detection of malignant 
lesions. The differences between various MRI sequences regarding lesion detection were compared using the Friedman test. Post hoc analysis with Wilcoxon signedrank tests was conducted with a Bonferroni correction applied. Survival analysis was performed using KaplanMeier curves with the day of diagnosis on imaging as entry date and log-rank test to test for statistical significance. A $p$ value of less than 0.05 was considered statistically significant.

\section{Results}

\section{Patients}

Sixty-six consecutive patients (median age 65 years, range 36-82 years) out of 93 patients with potentially resectable pancreatic cancer were eligible for inclusion. Twenty-seven patients were excluded for the following reasons: no confirmation of the presence or absence of malignant lesions $(n=4)$, local or systemic treatment prior to imaging $(n=3)$, artifacts or incomplete liver imaging
( $n=8)$, and a time interval between imaging or imaging and surgery of more than two months $(n=12)$. Nineteen (29\%) patients were diagnosed with liver metastases. Altogether 32 out of 47 patients without liver metastases underwent resection of the tumor. In the remaining 15 patients, the tumor was unexpectedly locally advanced $(n=12)$, metastasized intraperitoneally $(n=2)$, or the patient was too weak for surgery $(n=1)$. There was a significant difference in the survival between patients with liver metastases and without liver metastases $\left(\chi^{2}(2)=28.354, \quad p=0.000\right)$. Descriptives of included patients are described in Table 2.

\section{Confirmation of findings}

Confirmation of liver metastases was obtained by histopathology in twelve patients; only in two cases transabdominal ultrasound with biopsy was successful. In the remaining patients, histopathology was obtained intraoperatively $(n=9)$ or by autopsy $(n=1)$. In six patients without histological proof, preoperative ${ }^{18}$ FDG-PET showed avid lesions in the liver, suggestive of liver

Table 2 Descriptives

Liver metastases 19 (29\%) $\quad$ No liver metastases 47

Total population $66(100 \%) \quad p$ value (71\%)

\begin{tabular}{|c|c|c|c|c|}
\hline \multicolumn{5}{|l|}{ Gender } \\
\hline Men & $13(68 \%)$ & $29(62 \%)$ & $42(64 \%)$ & \\
\hline Women & $6(32 \%)$ & $18(38 \%)$ & $24(36 \%)$ & \\
\hline Age (years) & Median 64 (50-81) & Median 66 (36-82) & Median 65 (36-82) & 0.828 \\
\hline \multicolumn{5}{|l|}{ Primary tumor location } \\
\hline Head & $15(79 \%)$ & $37(79 \%)$ & $52(79 \%)$ & \\
\hline Body/tail & $2(11 \%)$ & $7(15 \%)$ & $10(15 \%)$ & \\
\hline Both & $1(5 \%)$ & $3(6 \%)$ & $4(6 \%)$ & \\
\hline Ca19.9 & $\begin{array}{l}\text { Median } 430(0-5297) \\
\quad(n=16)\end{array}$ & $\begin{array}{l}\text { Median } 155(1-7400) \\
\quad(n=42)\end{array}$ & $\begin{array}{l}\text { Median } 191(0-7400) \\
\quad(n=58)\end{array}$ & 0.044 \\
\hline \multicolumn{5}{|l|}{ Tumor stage } \\
\hline I & - & - & - & \\
\hline II & - & $27(57 \%)$ & $27(41 \%)$ & \\
\hline III & - & $17(36 \%)$ & $27(26 \%)$ & \\
\hline IV & $19(100 \%)$ & $3(6 \%)$ & $27(33 \%)$ & \\
\hline \multicolumn{5}{|l|}{ Treatment } \\
\hline Curative resection & - & $32(68 \%)$ & $32(48 \%)$ & \\
\hline Palliative bypass & $7(37 \%)$ & $6(13 \%)$ & $13(20 \%)$ & \\
\hline Explorative laparotomy & $3(16 \%)$ & $5(11 \%)$ & $8(12 \%)$ & \\
\hline $\begin{array}{l}\text { Supportive care or palliative } \\
\text { chemotherapy }\end{array}$ & $9(47 \%)$ & $4(9 \%)$ & $13(20 \%)$ & \\
\hline Survival (weeks) & Median $18 \pm 1.9$ & Median $60 \pm 8.1$ & Median $47 \pm 3.0$ & 0.000 \\
\hline
\end{tabular}


metastases. In one patient multiple liver metastases were confirmed by intraoperative inspection and palpation of the liver and peritoneal metastases were histologically proven. The absence of liver metastases in the remaining 46 patients was confirmed intraoperatively by inspection and palpation of the liver $(n=43)$ and ${ }^{18}$ FDG-PET $(n=4)$. The mean time interval between CECT and Gd-enhanced MRI with DWI was 15 days (SD 12 days) and 26 days (SD 14 days) between Gd-enhanced MRI with DWI and surgery.

\section{Lesion analysis}

Gd-enhanced MRI with DWI detected malignant lesions in 16 out of 19 patients with liver metastases. The sensitivity of Gd-enhanced MRI with DWI was $84 \%$ (95\% CI $60-97 \%)$. The positive predictive value was $94 \%$ (95\% CI $69-99 \%)$, and the negative predictive value was $94 \%$ (95\% CI $85-98 \%$ ). There was one false positive on a per-patient basis, in this patient one liver lesion with perilesional ring enhancement and persistent high signal intensity on DWI was characterized as malignant on Gd-enhanced MRI with DWI. There was no evidence of liver metastases during surgery and follow-up CECT after 1 year. There were three false negatives on a per-patient basis. In the first case, one indeterminate lesion in liver segment six on CECT was characterized as benign on Gd-enhanced MRI with DWI. However, intraoperative biopsy-proven metastasis in segment two was not detected on MRI. In the second case, there were neither liver lesions on CECT nor Gd-enhanced MRI with DWI. In the last case, one lesion was indeterminate on Gd-enhanced MRI with DWI, yet showed high uptake on preoperative ${ }^{18}$ FDG-PET and thus was classified as metastasis.

In the negative-on-CT group, the per-patient prevalence of liver metastases was $20 \%$ (9/44). MRI was of additional value in $16 \%$ (7/44). In the indeterminate-on-CT group, the per-patient prevalence of liver metastases was $45 \%$ (10/ 22). MRI was of additional value in $90 \%$ of the patients $(20 / 22)$.

On a per lesion basis, Gd-enhanced MRI with DWI detected 397 malignant lesions in 16 out of 19 patients with liver metastases. Contrast-enhanced MRI detected 156 malignant lesions, whereas DWI detected 397 malignant lesions $(p=0.051)$. In three patients, 20 to 50 malignant lesions were detected only by DWI. In one patient, even more than 100 malignant lesions were visible only on DWI (Fig. 1). Table 3 summarizes the detection rate of malignant lesions in the different sequences of Gd-enhanced MRI with DWI. There was a statistically significant difference in the number of malignant lesions detected by T2W-HASTE, T1W-VIBE precontrast, arterial phase, portal-venous phase, and DWI $\left(\chi^{2}(2)=32.861\right.$, $p=0.000$ ). Post hoc analysis with Wilcoxon signed-rank tests was conducted with a Bonferroni correction applied, resulting in a statistically significant difference with a $p$ value of 0.005 . DWI detected significantly more metastases compared to T2W-HASTE $(Z=-3.181, p=0.001)$, T1W-VIBE precontrast $(Z=-3.183, p=0.001)$, arterial phase $(Z=-2.943, p=0.003)$, and portal-venous phase $(Z=-3.063, p=0.002)$. Figures 2,3 , and 4 show examples of three different patterns of liver metastases of pancreatic cancer on Gd-enhanced MRI with DWI.

Ninety-five percent of all liver metastases detected on Gd-enhanced MRI with DWI were subcentimeter lesions: $85 \% \leq 5 \mathrm{~mm}, 10 \% \quad 6-10 \mathrm{~mm}$, and $5 \%>10 \mathrm{~mm}$. Nine patients $(47 \%)$ had oligometastatic liver disease (i.e., $\leq 5$ liver metastases [29]) and eleven patients had polymetastatic liver disease.

\section{Discussion}

In this study, liver metastases were accurately diagnosed by Gd-enhanced MRI with DWI in 16 out of 66 (24\%) patients initially diagnosed with potentially resectable pancreatic cancer on CECT. Adding a diffusionweighted MRI to the contrast-enhanced MRI increased the number of detected metastases from 156 to 397. The combination of contrast-enhanced MRI and diffusionweighted MRI yielded a high detection rate in previous studies, particularly in small metastases [30]. Metastases of pancreatic cancer are mostly small and multiple, which is consistent with the study by Danet et al. [28], subcentimeter lesions comprising $95 \%$ of all lesions. DWI seems particularly useful in the estimation of the metastatic load with the detection of metastases that are smaller than $5 \mathrm{~mm}$.

The prevalence of liver metastases in this study was relatively high, 29\%. The reported prevalence of liver metastases in the previous studies varies from $4.9 \%$ to $30 \%$ [9, 17-20, 22]. Patients with borderline resectable tumors and patients with indeterminate liver lesions were included, with a higher probability of having liver metastases. Additionally, on Gd-enhanced MRI there were metastases with a hypervascular enhancement pattern. A CECT with only porto-venous phase might have decreased the detection of these hypervascular metastases and overall the ability to characterize focal liver lesions on CECT. These factors might attribute to the higher additional value of MRI in this study as compared to the previous studies. The sensitivity of combined contrast-enhanced and diffusionweighted MRI was $84 \%$, which was comparable to other studies with sensitivities ranging from 73 to $100 \%$ $[9,17,18,22]$. Given the aggressiveness of pancreatic cancer and its tendency for rapid metastatic spread, 

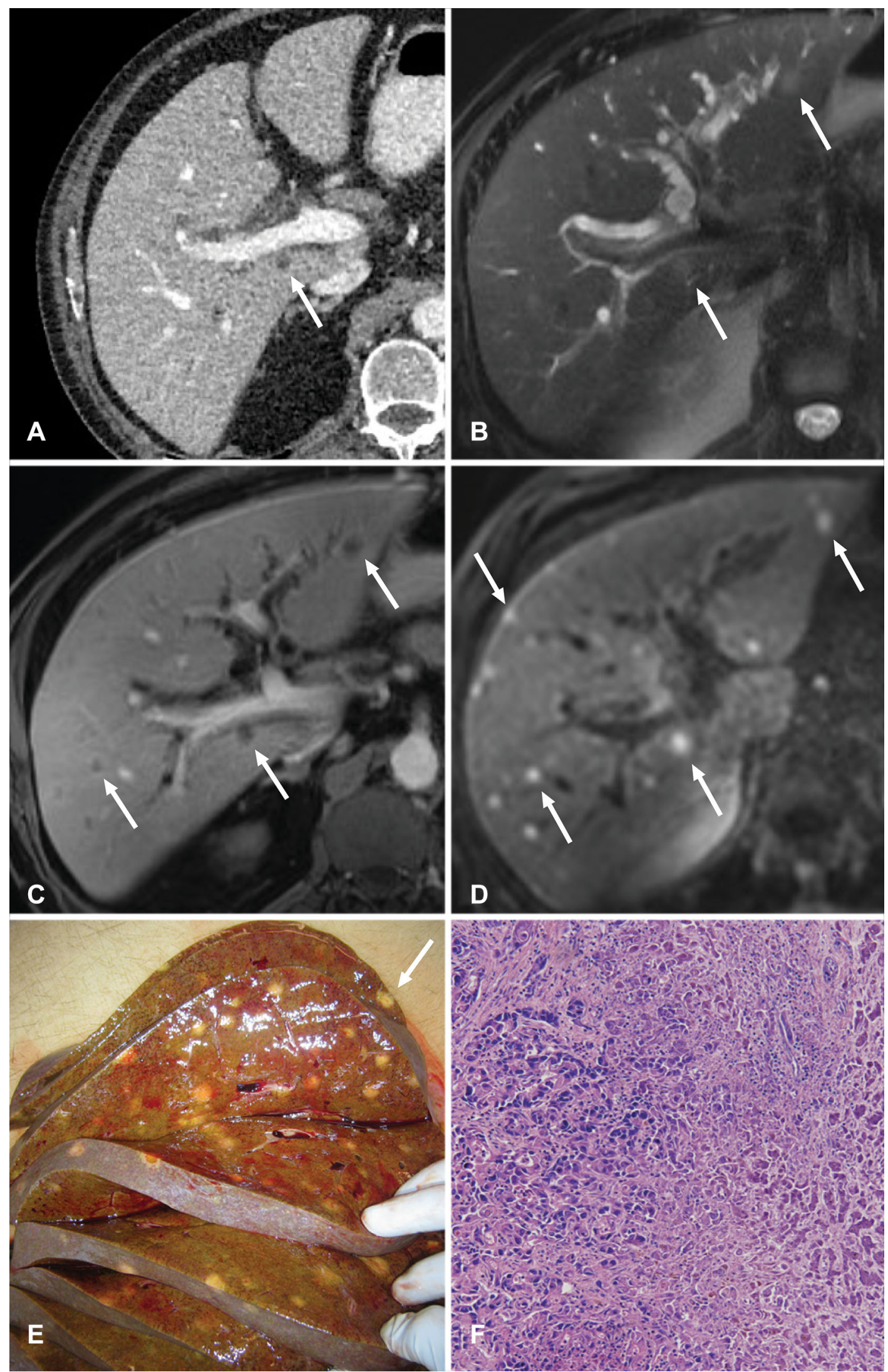
4Fig. 1 A 64-year-old male patient with borderline resectable pancreatic cancer on CECT and indeterminate liver lesions. The lesions were characterized as liver metastases by Gd-enhanced MRI. DWI additionally showed $>100$ metastases. The time interval between CT and MRI was 11 days. In this patient, there was a large discrepancy between CECT and Gd-enhanced MRI and DWI. Within 4 weeks after initial diagnosis, the patient died of cholangitis septicemia. An autopsy was performed and confirmed MRI findings of more than 100 liver metastases. CECT (a) shows multiple hypodense liver lesions too small to characterize. These lesions show moderately high signal intensity on T2W-HASTE (b), and post-contrast T1W-VIBE portalvenous phase (c) shows rim enhancement. Diffusion-weighted MRI shows multiple lesions (white arrows) with a high signal intensity that remain hyperintense on the high $b$-value $b=800 \mathrm{~s} / \mathrm{mm}^{2}$ (d). The autopsy confirmed there were more than 100 liver metastases (E\&F)

differences in sensitivity might be caused by differences in the time interval between Gd-enhanced MRI with DWI and the reference standard. The mean time interval in this study between CECT and Gd-enhanced MRI with DWI was 15 days and 26 days between Gd-enhanced MRI with DWI and surgery. A time interval of less than 20-25 days between imaging and any planned definitive therapy seems appropriate to grant accurate staging [4, 5, 31, 32]. Observer bias might have influenced the results of the study in favor of Gd-enhanced MRI with DWI, as only one observer re-evaluated the images, although in routine clinical practice images are also viewed by one observer, and the reported interobserver agreement for focal liver lesions in previous studies was good to excellent $[15,17,18,33-35]$.

A major problem was histopathological confirmation of the findings on Gd-enhanced MRI with DWI, as biopsy of all liver lesions is not possible and unethical in a living patient. Therefore, determining diagnostic accuracy on a per lesion basis is nearly impossible. Moreover, in our
Table 3 Number of malignant lesions on different sequences of Gd-enhanced MRI with DWI

\begin{tabular}{llcccc}
\hline Sequence & T2W-MRI & T1W-MRI precontrast & T1W-MRI arterial & T1W-MRI portal-venous & DWI \\
\hline$\leq 5 \mathrm{~mm}$ & 13 & 9 & 100 & 90 & 339 \\
$6-10 \mathrm{~mm}$ & 30 & 27 & 32 & 30 & 38 \\
$>10 \mathrm{~mm}$ & 20 & 18 & 20 & 20 & 20 \\
Total & 63 & 54 & 152 & 140 & 397 \\
\hline
\end{tabular}

The number of suspected liver metastases on various sequences of Gd-enhanced MRI with DWI in patients with liver metastases

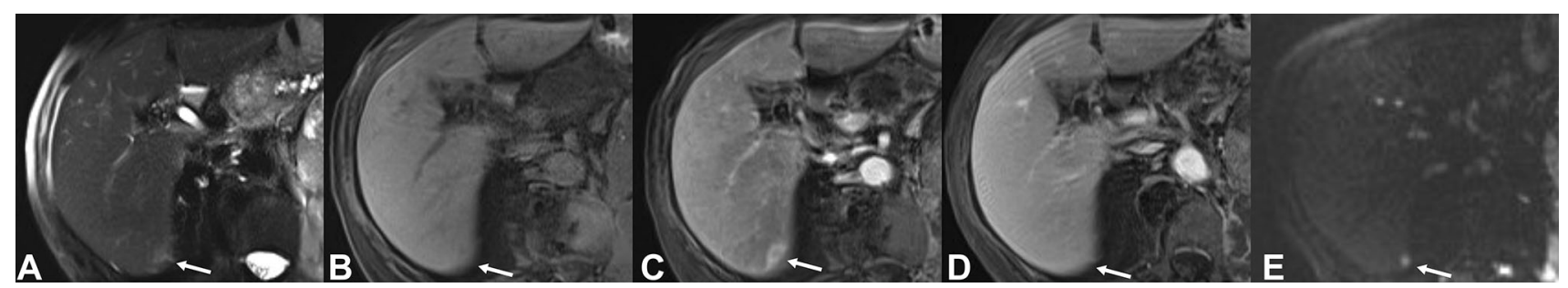

Fig. 2 A 70-year-old female patient with borderline resectable pancreatic cancer with three indeterminate liver lesions on CECT. Seven lesions were characterized as liver metastases by Gd-enhanced MRI with DWI. Liver metastases were confirmed by intraoperative inspection and palpation of the liver. a-e Subcapsular hypervascular lesion in liver segment six (arrow). Near isointense on T2W-HASTE (a), near isointense on the T1W-VIBE precontrast images (b), hyperintense with wedge-shaped enhancement in the arterial phase (c), near isointense in the portal-venous phase (d). Persistent high signal intensity on DWI $\left(b=800 \mathrm{~s} / \mathrm{mm}^{2}\right)(\mathbf{e})$

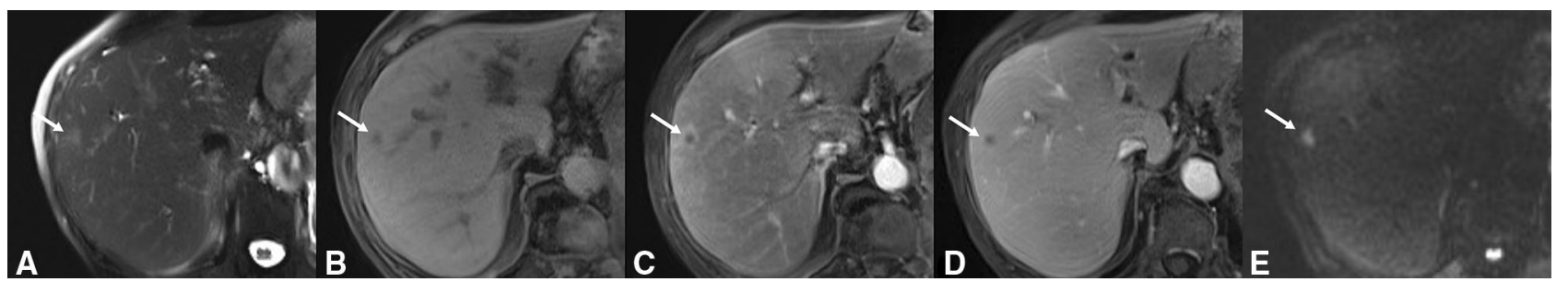

Fig. 3 This is the same patient as the patient in Fig. 2. a-e A malignant lesion with arterial perilesional ring enhancement with incomplete centripetal progression in liver segment eight (arrow). Moderately high on T2W-HASTE (a). Hypointense on T1W-VIBE precontrast (b), perilesional ring enhancement in the arterial phase with hypointense center $(\mathbf{c})$, which remains hypointense on the portalvenous phase (d). Persistent high signal intensity on DWI $(b=800 \mathrm{~s} /$ $\left.\mathrm{mm}^{2}\right)(\mathbf{e})$ 


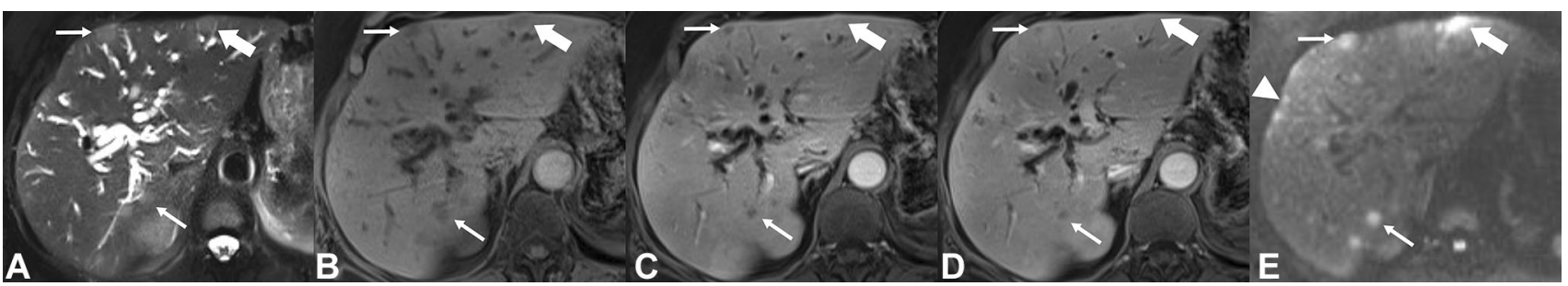

Fig. 4 A 53-year-old female patient with locally resectable pancreatic cancer on CECT. CECT showed indeterminate liver lesions, which were characterized as liver metastases by Gd-enhanced MRI with DWI. ${ }^{18}$ FDG-PET showed avid liver metastases and possible pulmonary metastasis. a-e Multiple capsular based and deep liver lesions with peripheral enhancement with complete or incomplete centripetal progression. Multiple malignant lesions with moderately

experience not all lesions on MRI are visible using either transabdominal or intraoperative ultrasound, therefore determining diagnostic accuracy on a per-patient basis remains challenging. In future clinical practice, MRI-guided biopsy with follow-up imaging could become an alternative strategy. In this study, there was one false positive on a per-patient basis; in previous studies false positives were also reported [17, 20, 22]. Therefore, at this moment we cannot deny patients surgery without histopathological proof of the radiological malignant liver lesions.

The increased safety of operations has led to more extensive local pancreas resections with venous and arterial reconstructions. Also, more effective chemotherapy protocols have been introduced, including combination therapies such as FOLFIRINOX. After neoadjuvant therapy in patients with borderline resectable pancreatic cancer or even locally advanced pancreatic cancer, secondary resection proved feasible with acceptable morbidity and survival rates [36]. Although still controversial, small studies and case reports have described select patients with oligometastatic hepatic metastases undergoing curative resection of the pancreas and the synchronous hepatic metastases $[37,38]$. To benefit from these developments, adequate staging is a prerequisite and information on size, number, and distribution of liver metastases are of the utmost importance. Improved detection of liver metastases could reduce futile resection of the tumor with its associated morbidity and mortality in these patients with a markedly reduced life expectancy. Moreover, it offers the possibility to start palliative systemic chemotherapy earlier as there is no recovery period from the operation. Also, it can reduce palliative bypass surgery as the prognosis for metastatic disease is even worse than for locally advanced disease [39]. Patients with obstructive symptoms can successfully be treated with endoscopically placed biliary and enteric stents, which is a safe, efficacious, and cost-effective procedure with good clinical outcome [40]. Finally, high signal intensity on T2W-HASTE (a), low signal intensity on T1W-VIBE precontrast (b), incomplete progression in the arterial (c), and portal-venous phase (thin arrows) (d). Another malignant lesion with complete progression to isointense enhancement in the portalvenous phase (thick arrow) (d). Persistent high signal intensity on DWI $\left(b=800 \mathrm{~s} / \mathrm{mm}^{2}\right)$. Some capsular lesions are only visible on DWI (arrowhead) (e)

improved detection of liver metastases during monitoring of (neo)adjuvant treatment could lead to a change in therapeutic strategy.

The retrospective nature of this study prevents a reliable calculation of the specificity, positive and negative predictive value of Gd-enhanced MRI with DWI versus CECT. Therefore, we started a large international multicenter prospective study to validate these results and to determine the diagnostic accuracy, implications for clinical decision making, and cost-effectiveness of Gd-enhanced MRI with DWI.

This study showed that Gd-enhanced MRI with DWI detected synchronous liver metastases in $24 \%$ of patients with potentially resectable pancreatic cancer on CECT with a sensitivity of $84 \%$. Contrast-enhanced MRI showed 156 malignant lesions versus 397 malignant lesions with DWI, most of which were particularly small $(<5 \mathrm{~mm})$.

Funding The authors state that this work has not received any funding.

\section{Compliance with ethical standards}

Conflict of interest The authors declare that they have no conflict of interest.

Open Access This article is distributed under the terms of the Creative Commons Attribution 4.0 International License (http://creative commons.org/licenses/by/4.0/), which permits unrestricted use, distribution, and reproduction in any medium, provided you give appropriate credit to the original author(s) and the source, provide a link to the Creative Commons license, and indicate if changes were made.

\section{References}

1. Siegel R, Ma J, Zou Z, Jemal A (2014) Cancer statistics, 2014. CA Cancer J Clin 64:9-29 
2. Rahib L, Smith BD, Aizenberg R, Rosenzweig AB, Fleshman JM, Matrisian LM (2014) Projecting cancer incidence and deaths to 2030: the unexpected burden of thyroid, liver, and pancreas cancers in the United States. Cancer Res 74:2913-2921

3. Are C, Chowdhury S, Ahmad H et al (2016) Predictive global trends in the incidence and mortality of pancreatic cancer based on geographic location, socio-economic status, and demographic shift. J Surg Oncol 114:736-742

4. Raman SP, Reddy S, Weiss MJ et al (2015) Impact of the time interval between MDCT imaging and surgery on the accuracy of identifying metastatic disease in patients with pancreatic cancer. AJR Am J Roentgenol 204:W37-42

5. Glant JA, Waters JA, House MG et al (2011) Does the interval from imaging to operation affect the rate of unanticipated metastasis encountered during operation for pancreatic adenocarcinoma? Surgery 150:607-616

6. Allen VB, Gurusamy KS, Takwoingi Y, Kalia A, Davidson BR (2016) Diagnostic accuracy of laparoscopy following computed tomography (CT) scanning for assessing the resectability with curative intent in pancreatic and periampullary cancer. Cochrane Database Syst Rev 7:Cd009323

7. Van den Broeck A, Sergeant G, Ectors N, Van Steenbergen W, Aerts R, Topal B (2009) Patterns of recurrence after curative resection of pancreatic ductal adenocarcinoma. Eur J Surg Oncol 35:600-604

8. Haeno H, Gonen M, Davis MB, Herman JM, Iacobuzio-Donahue CA, Michor F (2012) Computational modeling of pancreatic cancer reveals kinetics of metastasis suggesting optimum treatment strategies. Cell 148:362-375

9. HHolzapfel K, Reiser-Erkan C, Fingerle AA et al (2011) Comparison of diffusion-weighted MR imaging and multidetector-row $\mathrm{CT}$ in the detection of liver metastases in patients operated for pancreatic cancer. Abdom Imaging 36:179-184

10. Ducreux M, Cuhna AS, Caramella C et al (2015) Cancer of the pancreas: ESMO Clinical Practice Guidelines for diagnosis, treatment and follow-up. Annals of Oncology 26:v56-v68

11. Zins M (2015) Conventional imaging of pancreatic cancer. Rev Prat 65:376-378

12. Parsons CM, Sutcliffe JL, Bold RJ (2008) Preoperative evaluation of pancreatic adenocarcinoma. J Hepatobiliary Pancreat Surg 15:429-435

13. Eiber M, Fingerle AA, Brugel M, Gaa J, Rummeny EJ, Holzapfel K (2012) Detection and classification of focal liver lesions in patients with colorectal cancer: retrospective comparison of diffusion-weighted MR imaging and multi-slice CT. Eur J Radiol 81:683-691

14. Holzapfel K, Bruegel M, Eiber M et al (2010) Characterization of small $(</=10 \mathrm{~mm})$ focal liver lesions: value of respiratory-triggered echo-planar diffusion-weighted MR imaging. Eur J Radiol 76:89-95

15. Kim YK, Lee MW, Lee WJ et al (2012) Diagnostic accuracy and sensitivity of diffusion-weighted and of gadoxetic acid-enhanced 3-T MR imaging alone or in combination in the detection of small liver metastasis $(</=1.5 \mathrm{~cm}$ in diameter). Invest Radiol 47:159166

16. Hardie AD, Naik M, Hecht EM et al (2010) Diagnosis of liver metastases: value of diffusion-weighted MRI compared with gadolinium-enhanced MRI. Eur Radiol 20:1431-1441

17. Motosugi U, Ichikawa T, Morisaka H et al (2011) Detection of pancreatic carcinoma and liver metastases with gadoxetic acidenhanced MR imaging: comparison with contrast-enhanced multi-detector row CT. Radiology 260:446-453

18. Jeon SK, Lee JM, Joo I et al (2018) Magnetic resonance with diffusion-weighted imaging improves assessment of focal liver lesions in patients with potentially resectable pancreatic cancer on CT. Eur Radiol. https://doi.org/10.1007/s00330-017-5258-1
19. Chew C, O'Dwyer PJ (2016) The value of liver magnetic resonance imaging in patients with findings of resectable pancreatic cancer on computed tomography. Singapore Med J 57:334-338

20. Kim HW, Lee JC, Paik KH et al (2017) Adjunctive role of preoperative liver magnetic resonance imaging for potentially resectable pancreatic cancer. Surgery 161:1579-1587

21. Chang KJ, Kamel IR, Macura KJ, Bluemke DA (2008) 3.0-T MR imaging of the abdomen: comparison with $1.5 \mathrm{~T}$. Radiographics 28:1983-1998

22. Ito T, Sugiura T, Okamura $Y$ et al (2017) The diagnostic advantage of EOB-MR imaging over CT in the detection of liver metastasis in patients with potentially resectable pancreatic cancer. Pancreatology 17:451-456

23. Neri E, Bali MA, Ba-Ssalamah A et al (2016) ESGAR consensus statement on liver MR imaging and clinical use of liver-specific contrast agents. Eur Radiol 26:921-931

24. Talakic E, Steiner J, Kalmar P et al (2014) Gd-EOB-DTPA enhanced MRI of the liver: correlation of relative hepatic enhancement, relative renal enhancement, and liver to kidneys enhancement ratio with serum hepatic enzyme levels and eGFR. Eur J Radiol 83:607-611

25. Semelka RC, Brown ED, Ascher SM et al (1994) Hepatic hemangiomas: a multi-institutional study of appearance on T2weighted and serial gadolinium-enhanced gradient-echo MR images. Radiology 192:401-406

26. Bartolozzi C, Cioni D, Donati F, Lencioni R (2001) Focal liver lesions: MR imaging-pathologic correlation. Eur Radiol 11:13741388

27. Horton KM, Bluemke DA, Hruban RH, Soyer P, Fishman EK (1999) CT and MR imaging of benign hepatic and biliary tumors. Radiographics 19:431-451

28. Danet IM, Semelka RC, Nagase LL, Woosely JT, Leonardou P, Armao D (2003) Liver metastases from pancreatic adenocarcinoma: MR imaging characteristics. J Magn Reson Imaging 18:181-188

29. Hellman S, Weichselbaum RR (1995) Oligometastases. J Clin Oncol 13:8-10

30. Vilgrain V, Esvan M, Ronot M, Caumont-Prim A, Aube C, Chatellier G (2016) A meta-analysis of diffusion-weighted and gadoxetic acid-enhanced MR imaging for the detection of liver metastases. Eur Radiol 26:4595-4615

31. Sanjeevi S, Ivanics T, Lundell L et al (2016) Impact of delay between imaging and treatment in patients with potentially curable pancreatic cancer. Br J Surg 103:267-275

32. Healy GM, Redmond CE, Murphy S et al (2018) Preoperative CT in patients with surgically resectable pancreatic adenocarcinoma: does the time interval between CT and surgery affect survival? Abdom Radiol (NY) 43:620-628

33. Koh DM, Collins DJ, Wallace T, Chau I, Riddell AM (2012) Combining diffusion-weighted MRI with Gd-EOB-DTPA-enhanced MRI improves the detection of colorectal liver metastases. Br J Radiol 85:980-989

34. Lowenthal D, Zeile M, Lim WY et al (2011) Detection and characterisation of focal liver lesions in colorectal carcinoma patients: comparison of diffusion-weighted and Gd-EOB-DTPA enhanced MR imaging. Eur Radiol 21:832-840

35. Chung WS, Kim MJ, Chung YE et al (2011) Comparison of gadoxetic acid-enhanced dynamic imaging and diffusion-weighted imaging for the preoperative evaluation of colorectal liver metastases. J Magn Reson Imaging 34:345-353

36. Zins M, Matos C, Cassinotto C (2018) Pancreatic Adenocarcinoma Staging in the Era of Preoperative Chemotherapy and Radiation Therapy. Radiology 287:374-390

37. Lu F, Poruk KE, Weiss MJ (2015) Surgery for oligometastasis of pancreatic cancer. Chin J Cancer Res 27:358-367 
38. Klein F, Puhl G, Guckelberger O et al (2012) The impact of simultaneous liver resection for occult liver metastases of pancreatic adenocarcinoma. Gastroenterol Res Pract 2012:939350

39. Kneuertz PJ, Cunningham SC, Cameron JL et al (2011) Palliative surgical management of patients with unresectable pancreatic adenocarcinoma: trends and lessons learned from a large, single institution experience. J Gastrointest Surg 15:1917-1927

40. Yim HB, Jacobson BC, Saltzman JR et al (2001) Clinical outcome of the use of enteral stents for palliation of patients with malignant upper GI obstruction. Gastrointest Endosc 53:329-332 